\title{
Optimization of Smart Power Combining the Multiple Grids
}

\author{
Yichen Zhu ${ }^{a}$, Junxiang $\mathrm{Li}^{\mathrm{b},{ }^{*},}$, Yunjie $\mathrm{Xu}{ }^{\mathrm{c}}$, Li Ma ${ }^{\mathrm{d}}$ \\ Business School, University of Shanghai for Science and Technology, Shanghai 200093, China \\ afdssao@126.com, blijx@usst.edu.cn, c1172126122@qq.com, dmali5yue8@sina.com
}

Keywords: Smart grid, microgrid, energy-saving electricity.

\begin{abstract}
The smart use of electricity means combining coal-power grid with other multiple microgrids such as solar, wind and water power, and smartly distributing power through the user's power real-time information feedback in order to achieve low energy, low emission, high efficiency, green development and the power resources optimization. The proposed model can complement each other, recover and transfer excess power. The numerical result shows the superiority of intelligent power grid.
\end{abstract}

\section{Introduction}

Due to pollution problems and other reasons, a variety of new renewable energy sources such as wind, solar, water, tidal and nuclear energy, etc., are becoming more and more actively involved in the power system. It is widely regarded as one of the effective ways to connect the distributed powers[1] to the network in the form of microgrid[2]. As an intelligent and sustainable phase of power grid development, the third generation of power grids[3] has the features of supporting large-scale new-energy power supply, reducing substantially the security risk of interconnected large power grids and broadly integrating information and communication technologies, one of the important goals of which is to build large-scale new energy power transmission network[4-6].

This article focuses on the significance and intelligence of the microgrid and uses the model to analyze quantitatively the relationship between the members of the intelligent power grid and the superiority (Figure 1).

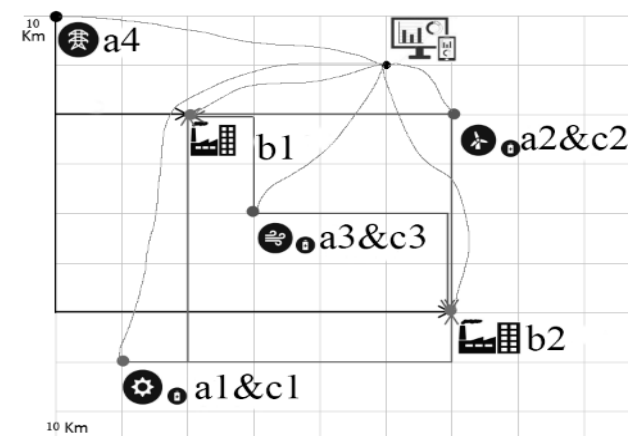

Fig. 1 Electricity delivery model

\section{Model Establishment and Simulation}

\subsection{Model Establishment}

Fig. 1 shows the basic working principle of grid intelligence through the economic cost optimization process of a specific model. The microgrid consists of the photovoltaic power plant a1, the wind power plant a2, the hydroelectric power station a3, the industrial region $\mathrm{b} 1$, the residential region b2 and their associated energy storage devices, converters, monitoring and protection devices, etc., which are connected with the large country power grid a4. The energy flow, shown as the straight lines in Fig. 1, represents the transmission of electric energy through cables and related physical facilities. The information flow, which is the curves in Fig. 1, represents the dispatch information of the demand side and supply side to the distributed automatic dispatching device for data analysis, and then the electricity energy is dispatched. 
The model is optimized by processing the corresponding data every hour of the day for a total of 24 times, so as to reflect the necessity and efficiency of the smart grid under the condition of the lowest cost. In this way, the mathematical model with the best economic cost under the combination of multiple networks is as follows:

$$
\begin{aligned}
& \operatorname{Min} \sum\left(p_{j} k_{c} l_{i j} x_{i j}+p_{j} x_{i j}\right)+k_{s}\left(c_{1}+c_{2}+c_{3}\right) \\
& (i=1,2 ; j=1,2,3,4)
\end{aligned}
$$

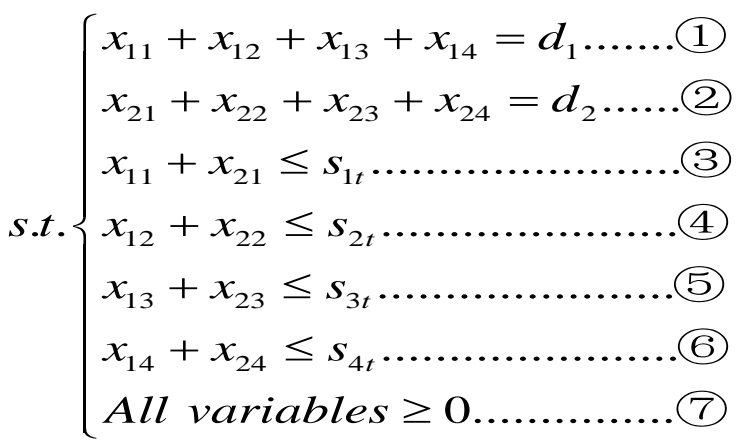

The explanation of the variables is listed Table 1.

Table 1 Explanation of the variables

\begin{tabular}{|c|c|c|}
\hline Name & Explanation & Variables \\
\hline Supply & The corresponding supply of power plants through the forecast \\
at different times & $s_{j t}$ \\
\hline Demand & The demand through the forecast at different times & $d_{j t}$ \\
\hline Actual cost & $\begin{array}{c}\text { Including production cost and corresponding environmental } \\
\text { remediation cost }\end{array}$ & $p_{j}$ \\
\hline Distance coefficient & Represent the distances between each power supply point & $l_{i j}$ \\
\hline $\begin{array}{c}\text { Supply situation } \\
\text { plan. }\end{array}$ & Phe actual supply situation and the various values required by this & $x_{i j}$ \\
\hline $\begin{array}{c}\text { Electricity storage cost } \\
\text { price }\end{array}$ & $\begin{array}{c}\text { Poss of energy per kilometer } \\
\text { quantity }\end{array}$ & $k_{s}$ \\
\hline $\begin{array}{c}\text { Electricity storage } \\
\text { Quantity that have not been allocated in the previous time period }\end{array}$ & $c_{j}$ \\
\hline Electricity loss rate & L & $k_{c}$ \\
\hline
\end{tabular}

The optimization goal is the optimal economic cost, which is composed of three parts, pj, kc and lij. xij is the transport loss. When the voltage of the cable is unchanged, the current will not change. Assuming that the temperature and transmission power of the power plant are constant, the circuit loss is approximately proportional to the length of the cable. pjxij is the actual cost of the corresponding electric energy and ks $(\mathrm{c} 1+\mathrm{c} 2+\mathrm{c} 3)$ is the excess storage cost. Constraints (1) and (2) indicate that the sum of the power output from the four power resource supply points to the each power consuming region should meet demand. Constraints (3), (4),(5) and (6) show that the sum of the power to the two power consuming region from the same power supply point will not exceed the supply capacity of the supply point. The last constraint (7) indicates that the supply demand is not negative under normal circumstances. 


\subsection{Data Input}

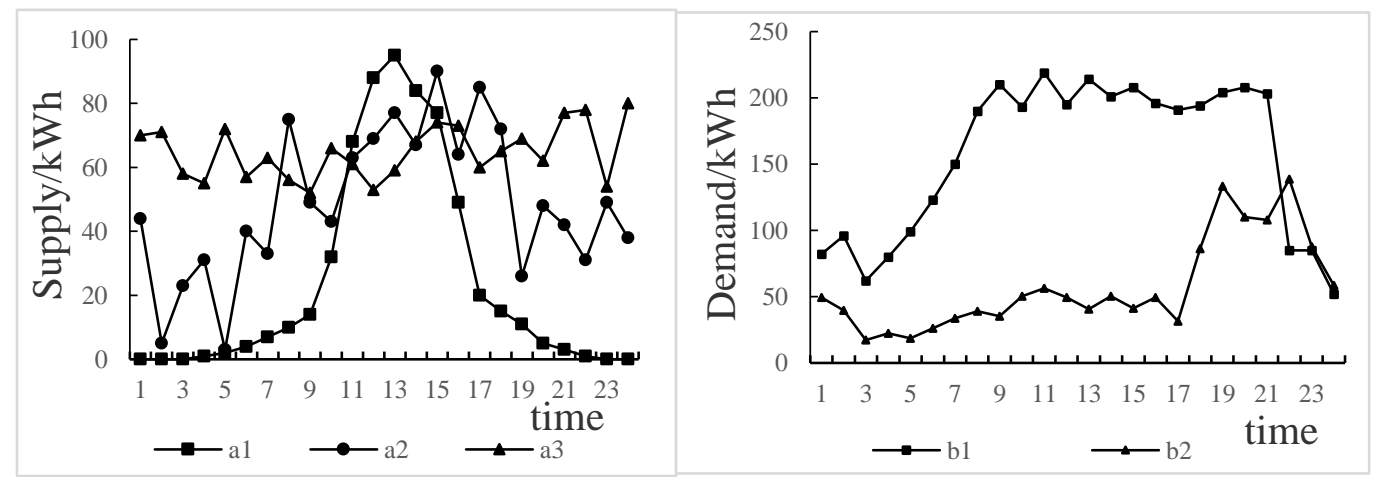

Fig. 2 Forecast supply and demand figures

Fig. 2 is the line chart of supply and demand for each power supply and consuming region predicted through data analysis. Among them, the photovoltaic power plant al generates electricity mainly in the daytime and achieves the highest efficiency from 12 to 14 . The wind power plant a2 generates the most electricity resources at 6 o'clock and 18 o'clock, and the hydroelectric power station a3 generates electricity stablely. The supply capacity of the large power grid a4 has always maintained a high value. Industrial region b1 demands highly electricity at 7 o'clock to 21 o'clock because the factories have a great deal of equipment in need of electricity and the power consumption begins to grow after rush hour in residential region $\mathrm{b} 2$.

\subsection{Simulation Result}

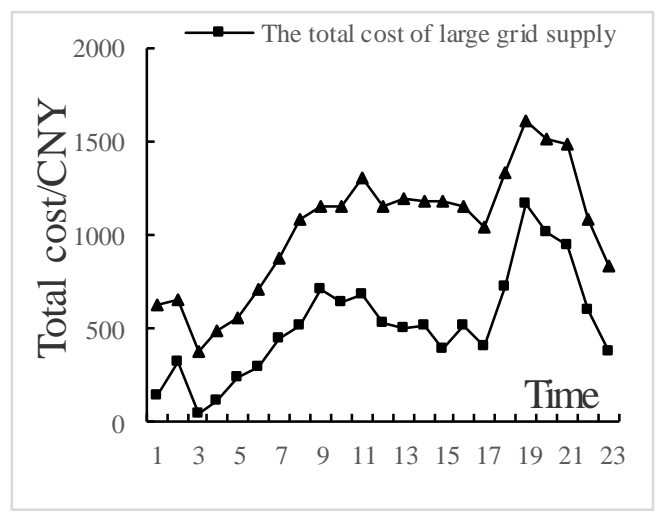

Fig. 3 Total cost curve

In the total cost curve (Fig. 3), the broken line marked with triangle is the total cost when only the large grid is used to meet the demand of the model. The broken line marked with square indicates an excellent solution under the combination of multiple grids.

Obviously, under the same abscissa, the value displayed by vertical axis of the triangle line chart is much larger than the square line chart, which means that the total cost under multigrid combination is much better than relying solely on the large power grid. Therefore, the use of multi-network combination of power supply will play a very prominent position in the future. At every time the power provided by power stations will reached the lowest cost under the premise of the highest utility, so the most intuitive effect of grid intelligence is that the energy efficiency will be improved significantly.

\subsection{Model Extension}

In order to be closer to the practical application, we will expand further the mathematical model. The first is the minimization of line losses, which is also the most fundamental role and one of the 
purpose of smart grids. Second, consideration should be given to reduce environmental impacts such as greenhouse gas emissions from diesel generators and micro-gas turbines and other pollutants emission. Benefiting from the rapid development of renewable energy technologies, the impact of new energy power generation on the environment has been greatly alleviated, compared with traditional power generation methods. In addition, it should be considered how to stabilize the voltage. Therefore, the following comprehensive objective function is considered:

$$
\operatorname{Min} F(x)=\rho 1 C(x)+\rho 2 E(x)+\rho 3 S(x)+\rho 4 O(x)
$$

Where $\mathrm{C}(\mathrm{x})$ is the economic constraint of configuration, which is expressed as the minimization of the path loss, $\mathrm{E}(\mathrm{x})$ is the environmental impact constraint which shows the size of the environmental pollution damage, $\mathrm{S}(\mathrm{x})$ is the voltage stability constraint and $\mathrm{O}(\mathrm{x})$ is the other existing constraint, such as resident satisfaction. $\rho 1, \rho 2, \rho 3$ and $\rho 4$ are the weights of each constraint.

\section{Summary}

Resource allocation and energy-saving optimization are the key parts of grid intelligence. It is of great significance to study how to establish the most efficient mathematical model, which plays a non-negligible role in building a complete new energy transportation network.

\section{Acknowledgments}

This research was sponsored by the National Natural Science Foundation of China (No. 71572113, 71432007), the matching project of NSFC (No. IP16303003, 2017KJFZ024, CFTD17004Z) and the Innovation Fund Project for Undergraduate Student of Shanghai (No. SH2016064, SH2017062, XJ2017090, XJ2017091, XJ20171105).

\section{References}

[1]. Weihua Luo, Wen Hu. The influence of the access of modern distributed power supply on the distribution network [J]. Technology Innovation and Application. 2017, (26):171-172.

[2]. Xinfa Yang, Jian Su, Zhipeng Lu, Haitao Liu, LI Rui. Overview on Micro-grid Technology [J]. Proceedings of the CSEE, 2014, 34(1): 57-70.

[3]. Shengwei Mei, Yuan Gong, Feng Liu. The Evolution Model of Three-generation Power Systems and Characteristic Analysis[J]. Proceedings of the CSEE, 2014, 34(7): 1003-1012.

[4]. Xiaoxin Zhou, Shuyong Chen, Zongxiang Lu. Review and Prospect for Power System Development and Related Technologies: a Concept of Three-generation Power Systems [J]. Proceedings of the CSEE, 2013, 36(22):1-11.

[5]. Guangyi Liu, Wendong Zhu, Jinxiang Chen, Yi Zhang. Characteristics, Application Scenarios and Analysis Platform of Smart Grid Big Data [J]. Southern Power System Techology, 2016, 10(05):102-110.

[6]. Dongxia Zhang, Xin Miao, Liping Liu, Yan Zhang, Keyan Liu. Research on Development Strategy for Smart Grid Big Data[J]. Proceedings of the CSEE, 2015, 35(1): 2-12. 